\title{
The usefulness of soluble receptor for advanced glycation end-products in the identification of COPD frequent exacerbator phenotype
}

This article was published in the following Dove Press journal: International Journal of COPD

\author{
Joanna Miłkowska- \\ Dymanowskal,2,* \\ Adam J Białas ${ }^{1,2, *}$ \\ Karolina Szewczyk' \\ Zofia Kurmanowska' \\ Paweł Górski ${ }^{1,2}$ \\ Wojciech J Piotrowski,2 \\ 'Department of Pneumology and \\ Allergy, Medical University of \\ Łódź, Łódź, Poland; ${ }^{2}$ Healthy Ageing \\ Research Centre, Medical University \\ of Łódź, Łódź, Poland \\ *These authors contributed equally \\ to this work
}

\begin{abstract}
Introduction: Exacerbations of COPD (ECOPDs) are important events in the course of COPD, accelerating the rate of decline in lung function and increasing the mortality risk. A growing body of evidence suggests the significance of the "frequent exacerbator" phenotype. This phenotype seems to be associated with a more severe airflow limitation, symptoms, health-related quality of life impairment, and higher mortality. However, there is no described biomarker that would help to identify this group of patients.

Patients and methods: Patients with COPD in "D" GOLD category were monitored for 3 years according to events of ECOPD. Serum samples were collected from the patients. Circulating level of plasma soluble receptor for advanced glycation end-products (sRAGE) was measured using commercially available high sensitivity kits. The receiver operating characteristic (ROC) curve analysis was used to assess the usefulness of sRAGE to identify frequent exacerbator phenotype. Log-rank test was used in the analysis of time to the subsequent exacerbation. Pearson $(R)$ or Spearman's rank $\left(R_{\mathrm{S}}\right)$ correlation coefficients were used for correlation analysis.
\end{abstract}

Results: Nineteen patients were enrolled. The area under the ROC curve (AUROC) for sRAGE for the identification of frequent exacerbator phenotype was 0.81 . Analysis identified the cutoff point as $850.407 \mathrm{pg} / \mathrm{mL}$, characterized by a sensitivity of 0.80 (95\% CI: $0.28-1.0)$ and specificity of 0.93 (95\% CI: $0.66-1.0$ ). Additionally, in the group with sRAGE $\leq 850.407 \mathrm{pg} / \mathrm{mL}$, we observed significantly shorter time to the subsequent exacerbation: median of 32 vs 105.5 days $(P=0.03)$. Correlation analysis revealed significant negative correlation between sRAGE and the number of exacerbations requiring hospitalization during the whole time of follow-up $\left(R_{\mathrm{S}}=-0.53 ; P=0.02\right)$ and significant positive correlation with $\mathrm{FEV}_{1}$ expressed as the percentage of reference value $(R=0.6 ; P=0.006)$.

Conclusion: sRAGE seems to be useful in the identification of frequent exacerbator phenotype. This parameter may also be used in the prediction of time to ECOPD. Our findings should be confirmed in a sufficiently powered larger sample.

Keywords: sRAGE, frequent exacerbator phenotype of COPD, exacerbations of COPD, COPD, prediction of AECOPD

\section{Introduction}

Exacerbations of COPD (ECOPDs) are key points for the natural history of COPD as they accelerate the rate of decline in lung function. ${ }^{1-3}$ Exacerbations requiring hospitalization are specifically important, because they are associated with significant mortality. In fact, evidence suggests that exacerbation with hypercapnia is burdened with significant in-hospital mortality. ${ }^{4}$ Additionally, long-term prognosis following hospitalization for ECOPD is burdened by the average in-hospital mortality rate of
Correspondence: Joanna MiłkowskaDymanowska

Department of Pneumology and Allergy, Medical University of Łódź, Kopcińskiego Street 22, Łódź 90-153, Poland Email joanna.milkowska-dymanowska@ umed.lodz.pl 
$6.7 \%$, while the average mortality rates at 3 and 6 months were reported to be 18 and $26 \%$, respectively, and those at 5 years were reported as high as $51 \% .^{5}$ The literature data show, that only $11.6 \%$ to $17 \%$ of COPD patients experience remittent exacerbations in 1 year. ${ }^{6-8}$ Nevertheless, this group induces an economic and social burden that is both substantial and increasing. ${ }^{3}$ This is why there is a strong need for elaborate approaches to prevent ECOPDs and identify in advance the group of subjects who experience remittent exacerbations. To gain this goal, there is a strong need for research for new, biochemical prediction tools.

The receptor for advanced glycation end-products (RAGE) is a multiligand, pattern-recognition receptor involved in the host response to injury, infection, and inflammation. ${ }^{9}$ It is an immunoglobulin superfamily protein of $35 \mathrm{kDa}$. Primarily, it plays a role of a multiligand transmembrane binder for several molecules. ${ }^{10}$ RAGE localizes in various cell types, such as monocytes, macrophages, smooth muscle cells, and endothelial cells. ${ }^{11}$ RAGE signaling also plays a key role in lung development and structure. ${ }^{12}$ Therefore, it justifies the linkage of RAGE with the pathobiology of COPD.

Numerous studies analyzed its role in cigarette smokeinduced airway inflammation and emphysema. ${ }^{12-16}$ Deficiencies in soluble RAGE (sRAGE) were linked to heightened inflammation in various chronic conditions, including neutrophilic airway inflammation in asthma and COPD. ${ }^{9}$ There is also an evidence for the usefulness of phenotyping of the RAGE gene, eg, the G82S polymorphism in the RAGE gene is associated with an increased risk of COPD, the GS genotype of the G82S variant is a risk factor for COPD in the Chinese population, ${ }^{17}$ and genetic variants in or near RAGE have also been associated with COPD affection status and emphysema, ${ }^{18-20}$ while the minor allele of the rs 2070600 variant was associated with protection with respect to COPD in the population of smokers. ${ }^{21}$ Moreover, RAGE was considered as a potential therapeutic target for COPD. ${ }^{22}$

We aimed to assess if sRAGE would be useful for the identification of patients prone to frequent ECOPDs. We also analyzed the time to the subsequent exacerbation and correlations between sRAGE and spirometry values.

\section{Patients and methods}

Study protocol was approved by Ethical Committee for Human Studies of the Medical University of Lodz. All patients provided written informed consent for the participation in the study. All study procedures were consistent with the tenets of the Declaration of Helsinki.
Patients with COPD in category D according to GOLD guidelines were enrolled to the study from July 2014 to August 2017. Patients were enrolled in the Outpatient Clinic and Department of Pneumology and Allergy, Medical University of Lodz. The main criterion was having $\geq 1$ exacerbation(s), which required hospitalization within last 12 months and/ or $\geq 2$ exacerbations not requiring hospitalization with the need for systemic steroid therapy and/or antibiotic therapy. All patients were treated with similar therapy. The exclusion criteria comprised a history of any active malignancy, $\alpha$-1-antitrypsin deficiency, HIV infection, and concomitant diagnosis of other significant respiratory diseases (eg, interstitial lung disease and parenchymal lung disease due to previous tuberculosis). Additionally, patients with exacerbations at least 8 weeks prior to sample collection were excluded from the study.

Serum samples were collected from the patients and stored at $-80^{\circ} \mathrm{C}$ until use. Repeated freeze-thaw cycles were avoided. ELISA was used to determine the serum levels of sRAGE (BioVendor - Laboratorni medicina a.s., Brno, Czech Republic) according to the manufacturer's recommendations.

Then, patients were monitored according to events of ECOPD by the same experienced physician (JM-D). Every such event was classified as mild (worsening of symptoms for 2 consecutive days and not treated with systemic corticosteroids and/or antibiotics), moderate (treated with systemic corticosteroids and/or antibiotics), and severe (requiring hospitalization) according to the Anthonisen criteria. Worsening of symptoms was captured in an electronic diary that alerted patients and physicians to the presence of an exacerbation. All events were confirmed by the physician. The current analysis focuses on moderate or severe exacerbations. The "frequent exacerbator" phenotype was defined by at least one hospitalization due to ECOPD or two treated at home per year in every year of prospective observation. The follow-up was terminated after 3 years in June 2018.

Continuous data were presented as the mean with SD or median with IQR, depending on the distribution of data. Variables were compared using the unpaired Student's $t$-test, Welch test, or the Mann-Whitney $U$-test, depending on data normality and the homogeneity of variance. Categorical data were presented as absolute value with percentage and were compared using Fisher's exact test.

The receiver operating characteristic (ROC) curve analysis was used to assess sRAGE usefulness to identify frequent exacerbator phenotype. The area under the ROC curve (AUROC) 
was presented as result and 95\% CI, while the accuracy of the qualitative test parameters was presented as point estimates and $95 \%$ CIs. An AUROC of greater than 0.9 was considered as excellent, greater than $0.8-0.9$ as very good, $0.7-0.8$ as good, $0.6-0.7$ as average, and below 0.6 as poor. ${ }^{23}$

Log-rank test was used in the analysis of time to the subsequent exacerbation. Pearson $(R)$ or Spearman's rank $\left(R_{\mathrm{S}}\right)$ correlation coefficients were evaluated to assess the relationships between sRAGE and spirometry values.

Data were analyzed using the R software for MacOS. ${ }^{24}$

\section{Results}

A total of 19 patients were included in the study. We identified five patients $(26.32 \%)$ who met the criteria of frequent exacerbator phenotype. The baseline clinical data and analysis after stratification by frequent exacerbator phenotype are presented in Table 1. As expected, frequent exacerbators experienced significantly higher number of all exacerbations during the time of follow-up: 5 (3-17) vs 1 (0.25-1.75); $P=0.002$. Additionally, in the nonfrequent exacerbator group, two patients were hospitalized because of ECOPD during the follow-up period (median 0) and the median of such exacerbations in the frequent exacerbator group was 2 (2-3).

ROC analysis showed an AUROC of 0.81 , which can be classified as very good accuracy according to Choi, ${ }^{23}$ and identified the cutoff point as $850.407 \mathrm{pg} / \mathrm{mL}$ (Figure 1), characterized by a sensitivity of 0.80 (95\% CI: 0.28-1.0), specificity of 0.93 (95\% CI: $0.66-1.0)$, positive predictive value (PPV) of 0.80 (95\% CI: 0.28-1.0), and negative predictive value (NPV) of 0.93 (95\% CI: 0.66-1.0). The diagnostic accuracy of the test was 0.9 (95\% CI: $0.67-0.99)$.

After stratification by the sRAGE cutoff point of 850.407 , we observed significantly shorter time to the subsequent exacerbation (median of 105.5 days for $\mathrm{sRAGE}>850.407 \mathrm{pg} / \mathrm{mL}$ and 32 days for sRAGE $\leq 850.407 \mathrm{pg} / \mathrm{mL}$ ) (Figure 2; $P$-value for log-rank test $=0.03$ ).

Correlation analysis revealed significant negative correlation between sRAGE and the number of exacerbations requiring hospitalization during the whole time of follow-up $\left(R_{\mathrm{S}}=-0.53\right.$; $P=0.02$ ) and significant positive correlation with $\mathrm{FEV}_{1}$ expressed as the percentage of reference value $(R=0.6 ; P=0.006)$. We did not observe significant correlation with $\mathrm{FVC}$ expressed as the percentage of reference value $(R=0.43 ; P=0.07)$.

Table I The baseline clinical data and analysis after stratification by frequent exacerbator phenotype

\begin{tabular}{|c|c|c|c|c|}
\hline Parameters & $\begin{array}{l}\text { Total } \\
(n=19)\end{array}$ & $\begin{array}{l}\text { Frequent } \\
\text { exacerbator } \\
(n=5)\end{array}$ & $\begin{array}{l}\text { Nonfrequent } \\
\text { exacerbator } \\
(n=\mid 4)\end{array}$ & $P$-value \\
\hline Age (years), mean (SD) & $66.32(7.06)$ & $63.6(5.08)$ & $67.29(7.56)$ & 0.33 \\
\hline Female, n (\%) & $14(73.68)$ & $5(100)$ & $9(64.29)$ & 0.26 \\
\hline History of COPD (years), mean (SD) & $10.21(7.83)$ & $10.4(3.21)$ & $10.14(9.04)$ & 0.95 \\
\hline History of smoking (pack-years), mean (SD) & $33.05(13.88)$ & $40.4(11.61)$ & $30.43(14.04)$ & 0.17 \\
\hline sRAGE $(\mathrm{pg} / \mathrm{mL})$, mean $(\mathrm{SD})$ & $975.68(399.31)$ & $657.89(437.88)$ & $1,089.18(330.25)$ & 0.03 \\
\hline $\mathrm{FEV}_{1}(\mathrm{~L})$, mean $(\mathrm{SD})$ & $0.91(0.49)$ & $0.54(0.17)$ & $\mathrm{I} .04(0.5 \mathrm{I})$ & 0.05 \\
\hline $\mathrm{FEV}_{1}(\%)$, mean (SD) & $36.6(17.11)$ & $23.72(5.91)$ & $41.21(17.55)$ & 0.005 \\
\hline FVC (L), mean (SD) & $1.94(0.88)$ & $1.48(0.53)$ & $2.11(0.94)$ & 0.09 \\
\hline FVC (\%), mean (SD) & $60.62(20.92)$ & $50.67(14.74)$ & $64.17(22.08)$ & 0.16 \\
\hline $\mathrm{FEV}_{\mathrm{I}} / \mathrm{FVC}$, mean (SD) & $0.46(0.12)$ & $0.37(0.05)$ & $0.49(0.13)$ & 0.07 \\
\hline mMRC, stage, median (IQR) & $3(2.25-4)$ & $4(3.5-4)$ & $3(2.25-3.75)$ & 0.28 \\
\hline BMI $\left(\mathrm{kg} / \mathrm{m}^{2}\right)$, mean $(\mathrm{SD})$ & $26.16(5.78)$ & $22.42(4.6)$ & $27.5(5.7)$ & 0.09 \\
\hline \multicolumn{5}{|l|}{ Major comorbidities ${ }^{\mathrm{a}}$} \\
\hline Ischemic heart disease, $\mathrm{n}(\%)$ & $8(42.11)$ & $3(60)$ & $5(35.7 I)$ & 0.6 \\
\hline Acute myocardial infarction in history, $\mathrm{n}(\%)$ & $4(21.05)$ & I (20) & $3(21.43)$ & 1.0 \\
\hline Congestive heart failure, $\mathrm{n}(\%)$ & $7(36.84)$ & $2(40)$ & $5(35.7 I)$ & 1.0 \\
\hline Arterial hypertension, $\mathrm{n}(\%)$ & $14(73.68)$ & $5(100)$ & $9(64.29)$ & 0.26 \\
\hline GERD, n (\%) & $5(26.32)$ & I (20) & $4(28.57)$ & 1.0 \\
\hline Obesity, n (\%) & $8(42.11)$ & I (20) & $7(50)$ & 0.34 \\
\hline
\end{tabular}

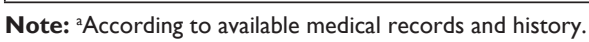

Abbreviation: sRAGE, soluble receptor for advanced glycation end-products. 


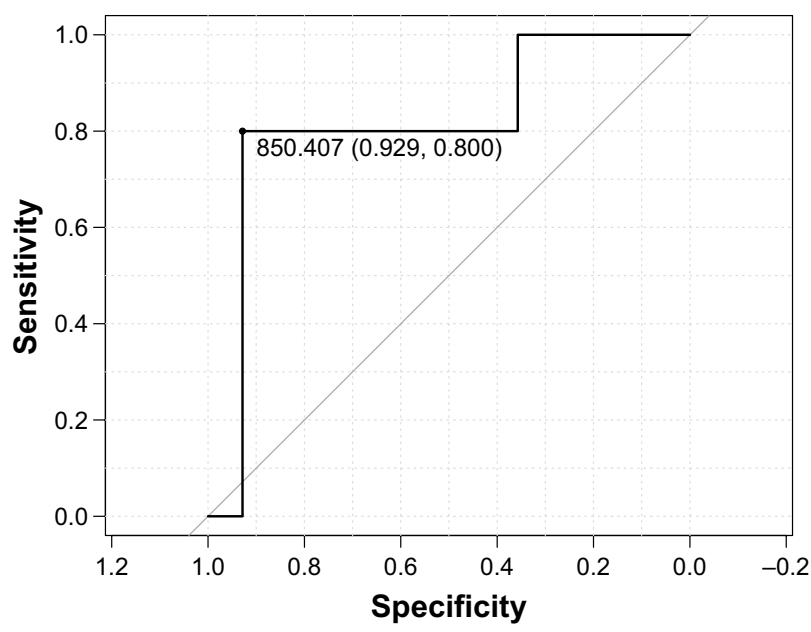

Figure I ROC curve for SRAGE in the identification of frequent exacerbator phenotype.

Note: The cutoff point presented best sum of specificity, and sensitivity presented in brackets was marked.

Abbreviations: ROC, receiver operating characteristic; sRAGE, soluble receptor for advanced glycation end-products.

\section{Discussion}

In modern treatment approaches, there is a tendency to identify relevant COPD phenotypes. It is believed that this may improve the precision, effectiveness, and safety of the treatment. Moreover, Golpe et $\mathrm{a}^{25}$ reported that grouping COPD patients according to clinical phenotypes can help us to identify those with a different mortality risk.

ECOPDs account for the greatest proportion of the total COPD burden on the health care system. Additionally, from $11.6 \%$ to $17 \%$ of COPD patients experience remittent exacerbations in 1 year. ${ }^{6-8}$ Also, changes in GOLD guidelines tend to extract patients who experience frequent exacerbations. ${ }^{3}$ Such approach enables early intensification of treatment to reduce the risk of future exacerbations in order to guide therapy. ${ }^{3}$

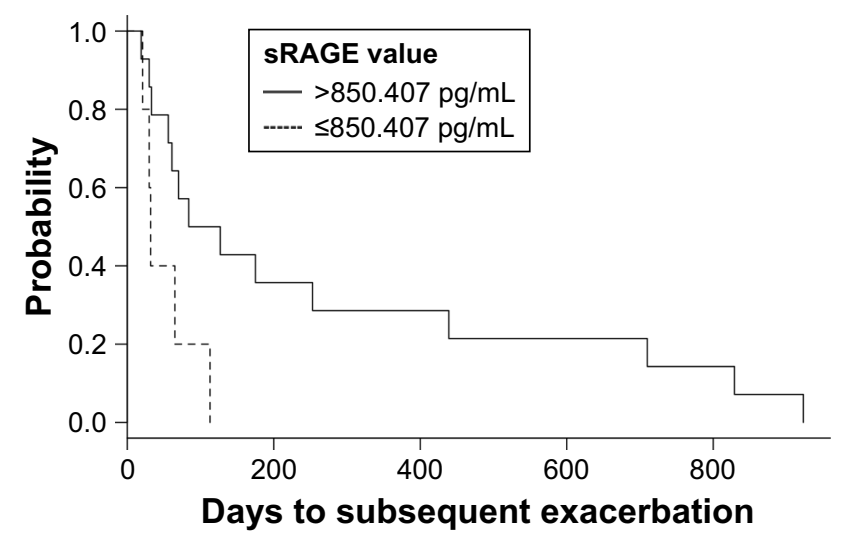

Figure 2 Kaplan-Meier curves for the time to the subsequent exacerbation (days), stratified by the sRAGE value of $850.407 \mathrm{pg} / \mathrm{mL}$.

Abbreviation: sRAGE, soluble receptor for advanced glycation end-products.
A growing body of evidence suggests the significance of the frequent exacerbator phenotype; however, there are no unified guidelines that defne this phenotype. Also, the knowledge on this group of patients seems to be scarce. The frequent exacerbator phenotype may be defined, concordantly with our study methods, as at least one hospitalization due to ECOPD or two treated at home per year. ${ }^{26}$ This phenotype seems to be associated with a more airflow limitation symptoms, health-related quality of life impairment, and higher mortality. ${ }^{26}$ Gupta et al ${ }^{27}$ reported the significant reduction in $\mathrm{FEV}_{1}$, diffusing capacity for carbon monoxide and transfer coefficient of the lung values in this group of patients.

There is no described biomarker that would help us to identify this group of patients. For this purpose, we propose sRAGE. ROC analysis showed very good diagnostic accuracy, and considering limitations of our pilot study, suggesting its potential usefulness. Additionally, we observed high values of accuracy of the qualitative test parameters.

Our finding is associated with potentially important clinical implications. Namely, identification of this group of patients would help us in planning more precise therapy in early stage of the disease. We believe that it will influence the natural history of the disease and, as the effect, ameliorate prognoses.

Moreover, sRAGE can also be used in the prediction of time to the subsequent exacerbation. This observation is of a great benefit for clinical practice because it would be potentially useful in indicating patients with a high risk of upcoming exacerbation and, thus, require early intensive treatment.

The parameter also correlated with $\mathrm{FEV}_{1}$, reflecting the relationship between decrease in $\mathrm{FEV}_{1}$ with increasing airway inflammation, which intensifies the progression of the disease. ${ }^{28}$

\section{Study limitations}

Small sample size is the major limitation of our study and determines this as a pilot study. Therefore, results should be considered as hypothesis generating and should be confirmed in a sufficiently powered larger sample. Small sample size of our study results from careful selection of the study group. All patients were treated by the same clinically experienced physician, and they were treated with similar therapy. The study group was observed for 3 years. Such studies are long lasting, time consuming, and involving deep cooperation with physicians. Additionally, our group consisted mainly of female subjects, which does not fully reflect overall COPD population. The female patients presented better compliance with the study protocol and more frequently expressed their 
consent for participation in the study. Moreover, there is an evidence that female COPD patients might be more prone to severe exacerbations, might a long period of symptoms before being admitted to the hospital, have higher number of hospitalizations, and prolonged length of hospital stay. ${ }^{29}$

\section{Conclusion}

sRAGE seems to be useful in the identification of frequent exacerbator phenotype. This parameter may also be used in the prediction of time to ECOPD. Our findings should be confirmed in a sufficiently powered larger sample.

\section{Acknowledgment}

The costs of this study were defrayed from regular finances of the Department of Pneumology and Allergy, Medical University of Lodz, Poland (503/1-151-03/503-11-001-17).

\section{Disclosure}

The authors report no conflicts of interest in this work.

\section{References}

1. Donaldson GC, Seemungal TA, Bhowmik A, Wedzicha JA. Relationship between exacerbation frequency and lung function decline in chronic obstructive pulmonary disease. Thorax. 2002;57(10):847-852.

2. Kanner RE, Anthonisen NR, Connett JE; Lung Health Study Research Group. Lower respiratory illnesses promote FEV(1) decline in current smokers but not ex-smokers with mild chronic obstructive pulmonary disease: results from the lung health study. Am J Respir Crit Care Med. 2001;164(3):358-364.

3. Global Initiative for Chronic Obstructive Pulmonary Disease. Global Strategy for the Diagnosis, Management, and Prevention of Chronic Obstructive Pulmonary Disease. Available from: https:/goldcopd.org/ wp-content/uploads/2017/11/GOLD-2018-v6.0-FINAL-revised-20Nov_WMS.pdf. Accessed August 21, 2018.

4. Connors AF, Dawson NV, Thomas C, et al. Outcomes following acute exacerbation of severe chronic obstructive lung disease. The SUPPORT investigators (Study to Understand Prognoses and Preferences for Outcomes and Risks of Treatments). Am J Respir Crit Care Med. 1996;154(4 Pt 1):959-967.

5. Hoogendoorn M, Hoogenveen RT, Rutten-van Mölken MP, Vestbo J, Feenstra TL. Case fatality of COPD exacerbations: a meta-analysis and statistical modelling approach. Eur Respir J. 2011;37(3):508-515.

6. Suissa S, Dell'aniello S, Ernst P. Long-term natural history of chronic obstructive pulmonary disease: severe exacerbations and mortality. Thorax. 2012;67(11):957-963.

7. Beeh KM, Glaab T, Stowasser S, et al. Characterisation of exacerbation risk and exacerbator phenotypes in the POET-COPD trial. Respir Res. 2013;14:116

8. Han MK, Quibrera PM, Carretta EE, et al. Frequency of exacerbations in patients with chronic obstructive pulmonary disease: an analysis of the SPIROMICS cohort. Lancet Respir Med. 2017;5(8):619-626.

9. Sukkar MB, Wood LG, Tooze M, et al. Soluble RAGE is deficient in neutrophilic asthma and COPD. Eur Respir J. 2012;39(3):721-729.

10. Mccance DR, Dyer DG, Dunn JA, et al. Maillard reaction products and their relation to complications in insulin-dependent diabetes mellitus. J Clin Invest. 1993;91(6):2470-2478.
11. Mukhopadhyay S, Mukherjee TK. Bridging advanced glycation end product, receptor for advanced glycation end product and nitric oxide with hormonal replacement/estrogen therapy in healthy versus diabetic postmenopausal women: a perspective. Biochim Biophys Acta. 2005; 1745(2):145-155.

12. Yonchuk JG, Silverman EK, Bowler RP, et al. Circulating soluble receptor for advanced glycation end products (sRAGE) as a biomarker of emphysema and the RAGE axis in the lung. Am J Respir Crit Care Med. 2015;192(7):785-792.

13. Chen M, Wang T, Shen Y, et al. Knockout of RAGE ameliorates mainstream cigarette smoke-induced airway inflammation in mice. Int Immunopharmacol. 2017;50:230-235.

14. Lee H, Park JR, Kim WJ, et al. Blockade of RAGE ameliorates elastaseinduced emphysema development and progression via RAGE-DAMP signaling. Faseb J. 2017;31(5):2076-2089.

15. Reynolds PR, Kasteler SD, Schmitt RE, Hoidal JR. Receptor for advanced glycation end-products signals through Ras during tobacco smokeinduced pulmonary inflammation. Am J Respir Cell Mol Biol. 2011; 45(2):411-418.

16. Robinson AB, Johnson KD, Bennion BG, Reynolds PR. RAGE signaling by alveolar macrophages influences tobacco smoke-induced inflammation. Am J Physiol Lung Cell Mol Physiol. 2012;302(11): L1192-L1199.

17. Li Y, Yang C, Ma G, et al. Association of polymorphisms of the receptor for advanced glycation end products gene with COPD in the Chinese population. DNA Cell Biol. 2014;33(4):251-258.

18. Castaldi PJ, Cho MH, San José Estépar R, et al. Genome-wide association identifies regulatory Loci associated with distinct local histogram emphysema patterns. Am J Respir Crit Care Med. 2014; 190(4):399-409.

19. Manichaikul A, Hoffman EA, Smolonska J, et al. Genome-wide study of percent emphysema on computed tomography in the general population. The Multi-Ethnic Study of Atherosclerosis Lung/SNP Health Association Resource Study. Am J Respir Crit Care Med. 2014;189(4):408-418

20. Cho MH, Castaldi PJ, Hersh CP, et al. A Genome-Wide Association Study of Emphysema and Airway Quantitative Imaging Phenotypes. Am J Respir Crit Care Med. 2015;192(5):559-569.

21. Young RP, Hay BA, Hopkins RJ. Does RAGE protect smokers from COPD? Eur Respir J. 2011;38(3):743-744.

22. Chen L, Liu L, Wang T, Shen YC, Wen FQ. Receptor for advanced glycation end products: a new theraputic target for chronic obstructive pulmonary disease? Arch Med Res. 2013;44(1):75-76.

23. Choi BC. Slopes of a receiver operating characteristic curve and likelihood ratios for a diagnostic test. Am J Epidemiol. 1998;148(11): 1127-1132.

24. R Core Team. R: A Language and Environment for Statistical Computing. R Foundation for Statistical Computing: Vienna, Austria; 2016.

25. Golpe R, Suárez-Valor M, Martín-Robles I, et al. Mortality in COPD patients according to clinical phenotypes. Int J Chron Obstruct Pulmon Dis. 2018;13:1433-1439.

26. Le Rouzic O, Roche N, Cortot AB, et al. Defining the "Frequent Exacerbator" Phenotype in COPD: A Hypothesis-Free Approach. Chest. 2018;153(5):1106-1115.

27. Gupta PP, Govidagoudar MB, Yadav R, Agarwal D. Clinical and pulmonary functions profiling of patients with chronic obstructive pulmonary disease experiencing frequent acute exacerbations. Lung India. 2018;35(1):21-26.

28. Oh JY, Sin DD. Lung inflammation in COPD: why does it matter? F1000 Med Rep. 2012;4:23.

29. Blumenthal JA, Emery CF, Smith PJ, et al. The effects of a telehealth coping skills intervention on outcomes in chronic obstructive pulmonary disease: primary results from the INSPIRE-II study. Psychosom Med. 2014;76(8):581-592. 


\section{Publish your work in this journal}

The International Journal of COPD is an international, peer-reviewed journal of therapeutics and pharmacology focusing on concise rapid reporting of clinical studies and reviews in COPD. Special focus is given to the pathophysiological processes underlying the disease, intervention programs, patient focused education, and self management protocols.

This journal is indexed on PubMed Central, MedLine and CAS. The manuscript management system is completely online and includes a very quick and fair peer-review system, which is all easy to use. Visit http://www.dovepress.com/testimonials.php to read real quotes from published authors. 